\title{
Liquid-Phase Exfoliation of Poly(Dicarbon Monofluoride) $\left(C_{2} F\right)_{n}$
}

Xianjue Chen, ${ }^{\mathrm{a},{ }^{*}}$ Marc Dubois,,${ }^{\mathrm{b},}$ Silvana Radescu, ${ }^{\mathrm{c}}$ Aditya Rawal, ${ }^{\mathrm{d}}$ Chuan Zhao ${ }^{\mathrm{a}}$

${ }^{a}$ School of Chemistry, University of New South Wales, Sydney, New South Wales 2052, Australia,

${ }^{b}$ Université Clermont Auvergne, SIGMA Clermont, CNRS, Institut de Chimie de Clermont-Ferrand (UMR 6296), BP 10448, F-63000, Clermont-Ferrand, France

${ }^{c}$ Departamento de Física, Universidad de La Laguna, Instituto de Materiales y Nanotecnología, 38200 La Laguna S/C Tenerife, Spain

${ }^{d}$ Mark Wainwright Analytical Centre, University of New South Wales, Sydney, New South Wales 2052, Australia

*Email: correspondence and requests for materials should be addressed to X.C. (xianjue.chen@unsw.edu.au) and M.D. (marc.dubois@uca.fr) 
Abstract: Fluorinated single-layer diamond ("F-diamond") is a new form of twodimensional (2D) carbon allotrope. Herein, poly(dicarbon monofluoride) $\left(\mathrm{C}_{2} \mathrm{~F}\right)_{\mathrm{n}}$ that is essentially made of stacked layers of "F-diamane" has been synthesized and exfoliated in a variety of solvents to yield well-dispersed ultrathin sheets. Microscopic and spectroscopic analyses revealed that the exfoliated sheets retained the "Fdiamane"-like structure. The experimental results are supported by the density functional theory (DFT) calculations.

Atomically thin diamond ("diamane") has recently emerged as a new twodimensional carbon allotrope. Theoretical studies first predicted the conversion of AB-stacked graphene few-layers into diamane films through the formation of sufficient carbon-fluorine (C-F) (or carbon-hydrogen $(\mathrm{C}-\mathrm{H})$ ) bonds on the two free surfaces. ${ }^{[1-4]}$ Odkhuu et al. showed that when a one-side surface of a bilayer graphene grown on a transition metal surface is fluorinated, the energetics for the conversion to $\mathrm{sp}^{3}$-bonded films were significantly lower than that for the free-standing bilayer. ${ }^{[5]}$ Fluorination and hydrogenation of graphene have been experimentally explored. ${ }^{[6,7]}$ Recent advances in the chemical vapor deposition (CVD) growth of graphene towards precisely controlling the quality of graphene (number of layers, crystallinity, coverage, uniformity) have suggested pathways for conversion of few layers of graphene into "diamane" films. ${ }^{[8]}$ Bakharev et al. ${ }^{[9]}$ first demonstrated that fluorine chemisorption on a CVD-grown AB-stacked bilayer graphene led to a fluorinated diamond monolayer ("F-diamane"). Rajasekaran et al. ${ }^{[10]}$ also reported experimental evidence that hydrogen adsorption could induce partial phase transition of few-layer graphene to a $\mathrm{sp}^{3}$-bonded structure on $\operatorname{Pt}(111)$ surface.

Fluorination of graphite was first reported in 1934 through exposing graphite to fluorine gas at moderate temperatures $\left(400-600{ }^{\circ} \mathrm{C}\right) .{ }^{[11]}$ The product is a solid fluorocarbon compound, which has been loosely classified as a graphite intercalation compound given its layered structures derived from graphite. ${ }^{[12]}$ By controlling the fluorination process, two forms of graphite fluoride can be obtained: "stage-1" $(\mathrm{CF})_{\mathrm{n}}$, i.e. fluorine atoms are bonded above and under graphene planes alternatingly and every carbon atom is covalently bonded with a fluorine atom, taking a chair-type structure, and "stage-2" $\left(\mathrm{C}_{2} \mathrm{~F}\right)_{\mathrm{n}}$, i.e. fluorine atom is inserted into every second layer 
of graphite and carbon atoms establish a $\mathrm{sp}^{3}$ orbital with a double-decked structure. The structural model proposed in previous studies ${ }^{[13-15]}$ is illustrated in Fig. 1a, b. Note that the $\left(\mathrm{C}_{2} \mathrm{~F}\right)_{\mathrm{n}}$ is essentially composed of stacked layers of "F-diamane". The low surface energy caused by the strong and short C-F bonds results in a low friction coefficient between the layers, ${ }^{[16]}$ and thus the "F-diamane" layers could be exfoliated under shear.

Liquid-phase exfoliation has been widely used to prepare single-layer and few-layer platelets from bulk flakes of layered materials including graphite, hexagonal boron nitride, black phosphorus, transition metal dichalcogenides, etc. ${ }^{[17-20]}$ Note that $(C F)_{n}$ has been exfoliated using different exfoliation methods. ${ }^{[21-27]}$ Unlike the CVD technique having a tight process control and relatively high cost, "top-down" exfoliation is a simple and versatile means to produce ultrathin layers in large quantity. ${ }^{28}$ Herein, we show that sonication-assisted exfoliation of $\left(\mathrm{C}_{2} \mathrm{~F}\right)_{\mathrm{n}}$ in solvents yields few-layer platelets with a "F-diamane"-like structure.

The synthesis of poly(dicarbon monofluoride) $\left(\mathrm{C}_{2} \mathrm{~F}\right)_{\mathrm{n}}$ was first reported in 1979. ${ }^{[13]}$ The yield of $\left(\mathrm{C}_{2} \mathrm{~F}\right)_{\mathrm{n}}$ depends on factors including the crystallinity of raw carbon, fluorinating reagent, and reaction temperature. Herein, the $\left(\mathrm{C}_{2} \mathrm{~F}\right)_{\mathrm{n}}$ was synthesized by heating graphite flakes to $350{ }^{\circ} \mathrm{C}$ under pure $\mathrm{F}_{2}$ gas flow (dynamic mode). SEM image shows the $\left(\mathrm{C}_{2} \mathrm{~F}\right)_{\mathrm{n}}$ flakes 5-20 $\mu \mathrm{m}$ in size, Fig. 1c. X-ray diffraction (XRD) pattern, Fig. 1d, suggests an interlayer distance of $\sim 0.81 \mathrm{~nm}$ corresponding to the (001) diffraction line of $\left(C_{2} F\right)_{n}$. The peak at $42.1^{\circ}$ is assigned to the (100) reflection $(d$ $=0.21 \mathrm{~nm}$ ). These values are in good agreement with the proposed structural model. The key feature distinguished from $(\mathrm{CF})_{\mathrm{n}}$ is that the $\left(\mathrm{C}_{2} \mathrm{~F}\right)_{\mathrm{n}}$ has a "stage-2" intercalated structure with a larger interlayer distance. In comparison, the XRD pattern (Fig. S1b) for $(\mathrm{CF})_{\mathrm{n}}$ suggests an interlayer distance of $0.64 \mathrm{~nm}$. The relatively broad peaks for the (001) and (100) reflections suggests a high degree of structural disorder in $\left(\mathrm{C}_{2} \mathrm{~F}\right)_{\mathrm{n}}$ and $(\mathrm{CF})_{\mathrm{n}}$. A peak at $26.6^{\circ}$ observed for both $\left(\mathrm{C}_{2} \mathrm{~F}\right)_{\mathrm{n}}$ and $(\mathrm{CF})_{\mathrm{n}}$ corresponds to the (002) diffraction from residual non-fluorinated graphitic structure.

The surface composition and C-F bonding of $\left(\mathrm{C}_{2} \mathrm{~F}\right)_{\mathrm{n}}$ were investigated using X-ray photoelectron spectroscopy (XPS). The survey spectrum (Fig. 2a) collected from $\left(\mathrm{C}_{2} \mathrm{~F}\right)_{\mathrm{n}}$ shows that it is mainly composed of $\mathrm{C}(60.2$ at.\%) and $\mathrm{F}$ (36.7 at.\%). The 
difference in the fluorine-to-carbon $(\mathrm{F} / \mathrm{C})$ ratio $\left(\mathrm{CF}_{0.61}\right.$ vs. $\left.\mathrm{CF}_{0.5}\right)$ could be owing to the presence of structural defects that allow carbon to be terminated with fluorine by forming $\mathrm{CF}_{2}$ and $\mathrm{CF}_{3}$ groups on the edges of the layers, and/or the presence of a small amount of $(\mathrm{CF})_{\mathrm{n}}{ }^{[13]}$ The XPS result for $(\mathrm{CF})_{\mathrm{n}}($ Fig. S1c) shows a significantly higher $\mathrm{F} / \mathrm{C}$ ratio of 1.0 .
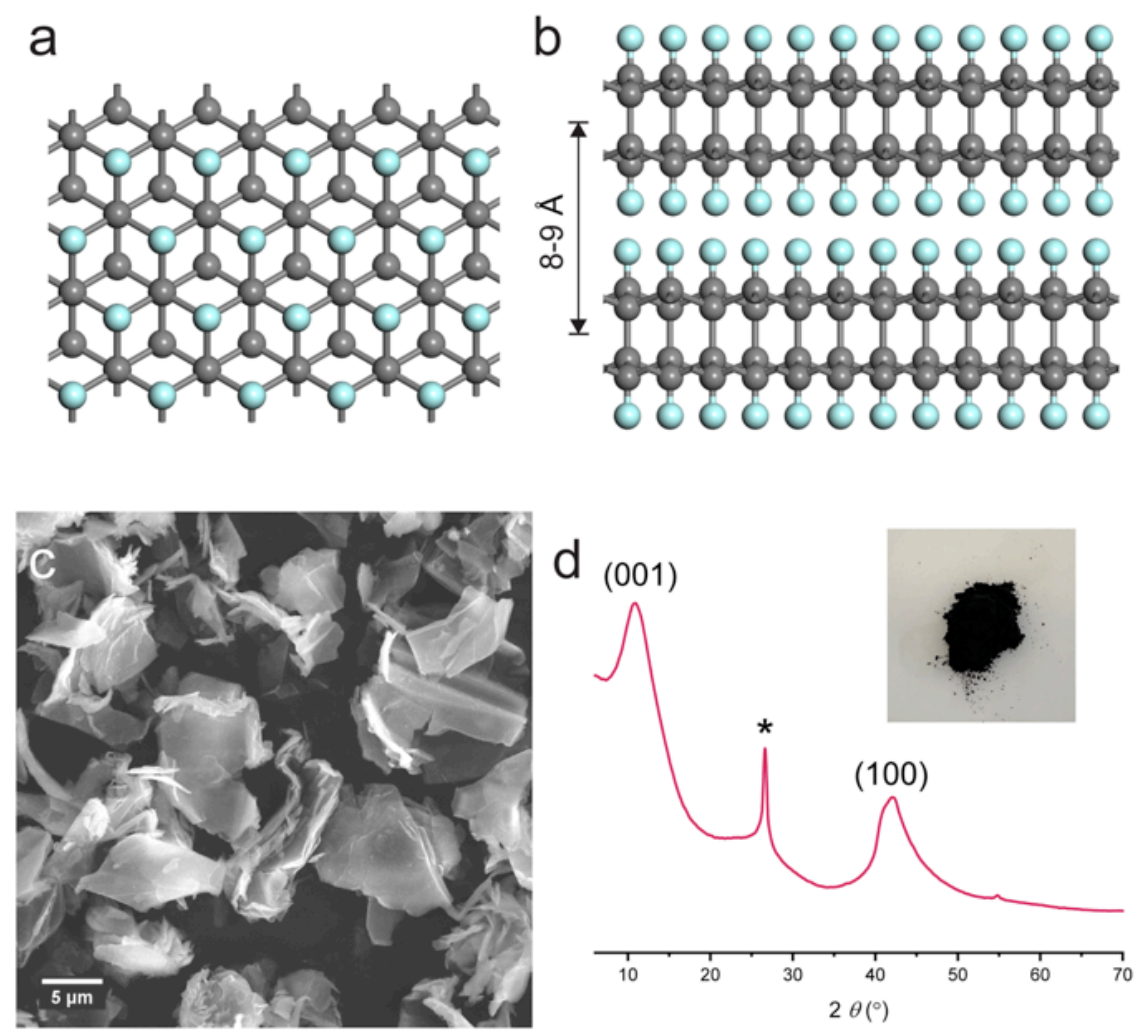

Fig. 1 The proposed (a) top-view and (b) side-view crystal structure of $\left(\mathrm{C}_{2} \mathrm{~F}\right)_{\mathrm{n}}$. (c) SEM image and (d) XRD pattern of $\left(\mathrm{C}_{2} \mathrm{~F}\right)_{\mathrm{n}}$ flakes. The inset shows a photograph of the $\left(\mathrm{C}_{2} \mathrm{~F}\right)_{\mathrm{n}}$ that has a dark grey color. The "**" symbols refer to the (002) diffraction from residual graphitic structure.

The high electronegativity of fluorine induces strong chemical shifts in the $\mathrm{C} 1 \mathrm{~s}$ binding energy, Fig. 2b. The $\mathrm{C} 1$ peak at $284.7 \mathrm{eV}$ is associated with the carbon atoms in the $\mathrm{sp}^{2}$ - and/or $\mathrm{sp}^{3}$-bonded structures; C2 $(285.7 \mathrm{eV})$ and C3 $(288.0 \mathrm{eV})$ peaks can be assigned to non-fluorinated carbons linked to fluorine bound carbons ( $\underline{\mathrm{C}}-\mathrm{CF}$ and CF-C-CF species); C4 peak $(289.7 \mathrm{eV})$ corresponds to the carbons forming $\mathrm{C}-\mathrm{F}$ bonds; $\mathrm{C} 5$ peak $(291.6 \mathrm{eV})$ is assigned to $\underline{\mathrm{CF}}_{2}$ groups that exist in the periphery of amorphous regions and/or platelet edges. ${ }^{[29]}$ Fig. S1d shows the C1s spectra of $(\mathrm{CF})_{\mathrm{n}}$, where the $\mathrm{C} 1, \mathrm{C} 2$, and $\mathrm{C} 3$ peaks have much lower relative intensities. This suggests 
that each fluorinated carbon in $(\mathrm{CF})_{\mathrm{n}}$ is predominantly bound to three other fluorinated carbons. The F1s peak (Fig. 2b and S1d) at $688.2 \mathrm{eV}$ is associated with covalently bound fluorine in both $\left(\mathrm{C}_{2} \mathrm{~F}\right)_{\mathrm{n}}$ and $(\mathrm{CF})_{\mathrm{n}}$.
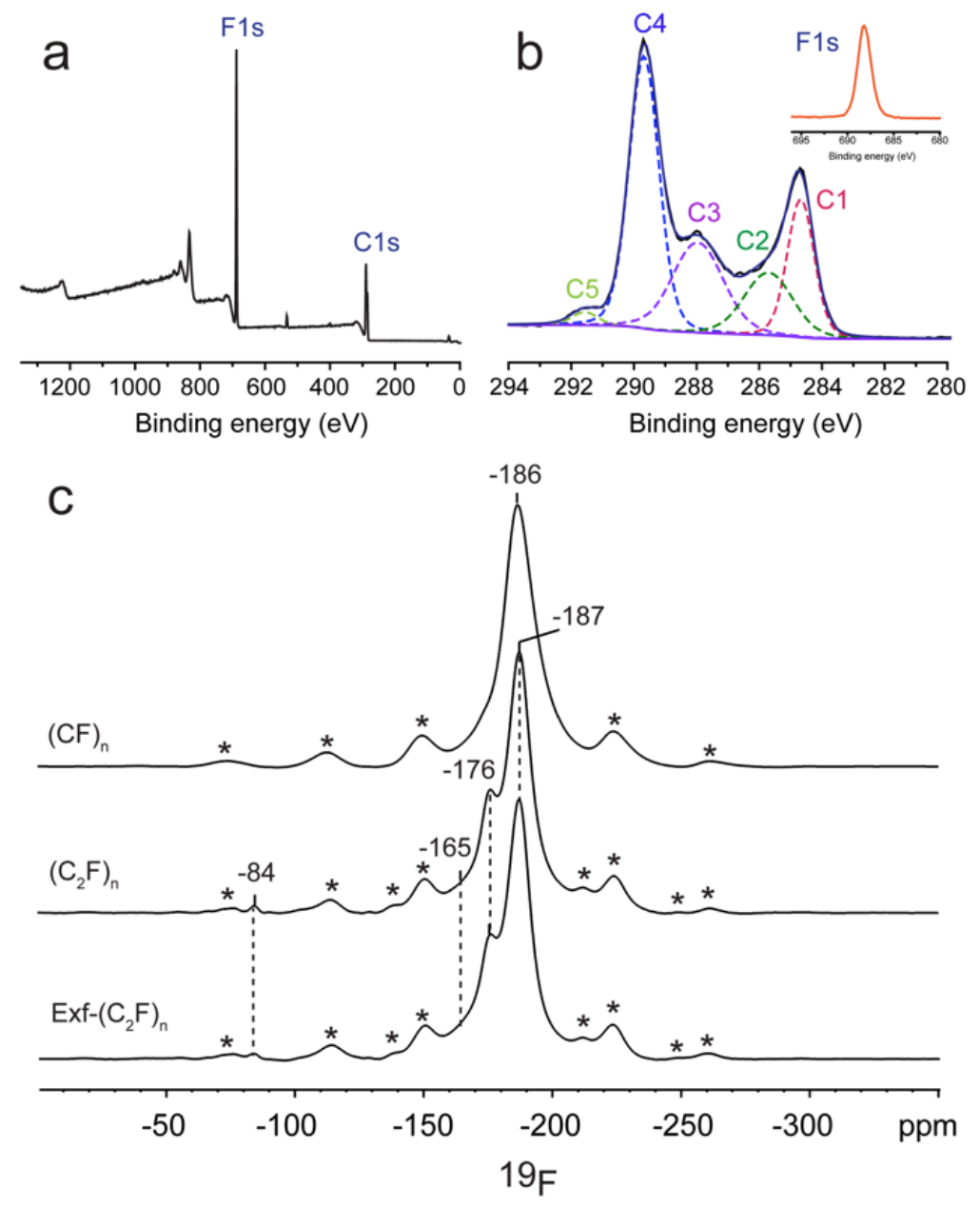

Fig. 2 XPS (a) survey and (b) $\mathrm{C} 1 \mathrm{~s}$ and $\mathrm{F} 1 \mathrm{~s}$ spectra for $\left(\mathrm{C}_{2} \mathrm{~F}\right)_{\mathrm{n}}$. (c) ${ }^{19} \mathrm{~F}$ Solid state NMR of $(\mathrm{CF})_{\mathrm{n}},\left(\mathrm{C}_{2} \mathrm{~F}\right)_{\mathrm{n}}$, and exfoliated $\left(\mathrm{C}_{2} \mathrm{~F}\right)_{\mathrm{n}}$.

Exfoliation of $\left(\mathrm{C}_{2} \mathrm{~F}\right)_{\mathrm{n}}$ within various solvents (water, ethanol, toluene, chloroform, and DMF) was carried out using probe sonication, Fig. S2. $\left(C_{2} F\right)_{n}$ can hardly be dispersed in water due to its hydrophobic nature, and most of the exfoliated material was floating on the water surface or sank to the bottom. Exfoliation within ethanol, chloroform, or DMF resulted in homogenous suspensions after removing any nondispersible species using centrifugation. Tyndall effect was observed for all the solvents (including water), suggesting the formation of colloidal dispersions of exfoliated $\left(\mathrm{C}_{2} \mathrm{~F}\right)_{\mathrm{n}}$. XPS spectra (Fig. S3) of the exfoliated $\left(\mathrm{C}_{2} \mathrm{~F}\right)_{\mathrm{n}}$ show increased $\mathrm{F} / \mathrm{C}$ ratios, $0.90,0.85,0.80,0.82,0.90$ for water, ethanol, toluene, chloroform, DMF. This 
could be owing to the exfoliation-induced fragmentation of $\left(\mathrm{C}_{2} \mathrm{~F}\right)_{\mathrm{n}}$ that exposes more edges where $\mathrm{CF}_{2}$ and $\mathrm{CF}_{3}$ groups are present, and/or conversion of $\left(\mathrm{C}_{2} \mathrm{~F}\right)_{\mathrm{n}}$ surface into $(\mathrm{CF})_{\mathrm{n}}$ due to partial defluorination.

Raman spectroscopy and Fourier-transform infrared spectroscopy (FTIR) analyses were carried out for $(\mathrm{CF})_{\mathrm{n}},\left(\mathrm{C}_{2} \mathrm{~F}\right)_{\mathrm{n}}$, and exfoliated $\left(\mathrm{C}_{2} \mathrm{~F}\right)_{\mathrm{n}}$, as shown in Fig. S1 and S4. For $(\mathrm{CF})_{\mathrm{n}}$, the Raman activity was quenched due to its wide band gap, ${ }^{[27]}$ whereas for $\left(\mathrm{C}_{2} \mathrm{~F}\right)_{\mathrm{n}}$ Raman bands associated with carbon D $\left(1324 \mathrm{~cm}^{-1}\right)$ and $\mathrm{G}\left(1586 \mathrm{~cm}^{-1}\right)$ bands were observed, which are close to the values measured in previous studies. ${ }^{[30]}$ In FTIR spectra, the strongest bands at $1192 \mathrm{~cm}^{-1}$ for $(C F)_{n}$ and at $1222 \mathrm{~cm}^{-1}$ for $\left(\mathrm{C}_{2} \mathrm{~F}\right)_{\mathrm{n}}$ can be assigned to the $\mathrm{C}-\mathrm{F}$ stretching vibration of tertiary carbon atoms. ${ }^{[31]} \mathrm{A}$ medium band at $1348 \mathrm{~cm}^{-1}$ was observed in the spectra, possibly corresponding to asymmetric stretching vibrations of peripheral $\mathrm{CF}_{2}$ groups. ${ }^{[32]}$ The exfoliated $\left(\mathrm{C}_{2} \mathrm{~F}\right)_{\mathrm{n}}$ show similar Raman and FTIR features to $\left(\mathrm{C}_{2} \mathrm{~F}\right)_{\mathrm{n}}$, which suggests that the "F-diamane" structure was retained.

Fig. 2c shows the ${ }^{19} \mathrm{~F}$ solid state NMR spectra of $(\mathrm{CF})_{\mathrm{n}},\left(\mathrm{C}_{2} \mathrm{~F}\right)_{\mathrm{n}}$, and exfoliated $\left(\mathrm{C}_{2} \mathrm{~F}\right)_{\mathrm{n}}$. The presence of covalent $\mathrm{C}-\mathrm{F}$ bonding in $\left(\mathrm{C}_{2} \mathrm{~F}\right)_{\mathrm{n}}$ and $(\mathrm{CF})_{\mathrm{n}}$ is confirmed by the position of the isotropic ${ }^{19} \mathrm{~F}$ NMR peaks. The primary signal of the ${ }^{19} \mathrm{~F}$ species was observed at the chemical shifts of $-186 \mathrm{ppm}$ and $-187 \mathrm{ppm}\left(\mathrm{vs} \mathrm{CFCl}_{3}\right)$, for the $(\mathrm{CF})_{\mathrm{n}}$ and $\left(\mathrm{C}_{2} \mathrm{~F}\right)_{\mathrm{n}}$ respectively. The $\left(\mathrm{C}_{2} \mathrm{~F}\right)_{\mathrm{n}}$ and the exfoliated $\left(\mathrm{C}_{2} \mathrm{~F}\right)_{\mathrm{n}}$ showed nearly identical ${ }^{19} \mathrm{~F}$ spectra, indicating that the exfoliation process did not induce major chemical changes in the material. A relatively weak signal at -84 ppm was observed for $\left(\mathrm{C}_{2} \mathrm{~F}\right)_{\mathrm{n}}$, corresponding to terminal $\mathrm{CF}_{3}$ groups. An associated isotropic signal relating to $\mathrm{CF}_{2}$ groups at $-120 \mathrm{ppm}$ is also expected, ${ }^{[33,34]}$ but in the present case is overlapped by spinning sidebands. A resolved shoulder at $-176 \mathrm{ppm}$ was observed in the $\left(\mathrm{C}_{2} \mathrm{~F}\right)_{\mathrm{n}}$, while a similar signal albeit with a lower resolution is also evident in $(\mathrm{CF})_{\mathrm{n}}$. This signal is typical of $\left(\mathrm{C}_{2} \mathrm{~F}\right)_{\mathrm{n}}$ layers, ${ }^{[33,34]}$ corresponding to the stacking of $\mathrm{F}-\mathrm{C}\left(\mathrm{sp}^{3}\right)$ $\mathrm{C}\left(\mathrm{sp}^{3}\right)$-F. Note that in the extreme case, such a chemical shift is close to fluorinated nanodiamonds, i.e. $\mathrm{C}\left(\mathrm{sp}^{3}\right)-\mathrm{C}\left(\mathrm{sp}^{3}\right)-\mathrm{F}$, with $\mathrm{F}$ being located only on the surface with low content (-164 ppm). ${ }^{[35]}$ Interestingly, deconvolution of the ${ }^{19} \mathrm{~F}$ spectra (Fig S5) indicates that the $\left(\mathrm{C}_{2} \mathrm{~F}\right)_{\mathrm{n}}$ show a relatively broad signal near $-168 \mathrm{ppm}$ suggesting the formation of such ${ }^{19} \mathrm{~F}$ species as well. 

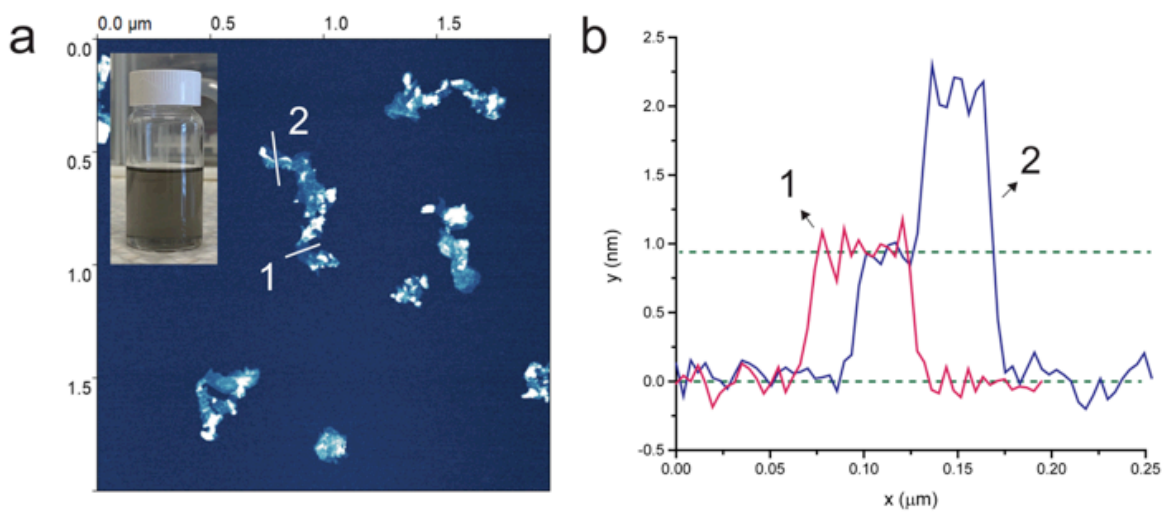

Fig. 3 (a) AFM image of exfoliated $\left(\mathrm{C}_{2} \mathrm{~F}\right)_{\mathrm{n}}$ platelets, with (b) the corresponding height analyses along the lines indicated in (a). A colloidal suspension containing exfoliated $\left(\mathrm{C}_{2} \mathrm{~F}\right)_{\mathrm{n}}$ platelets is shown in the inset.

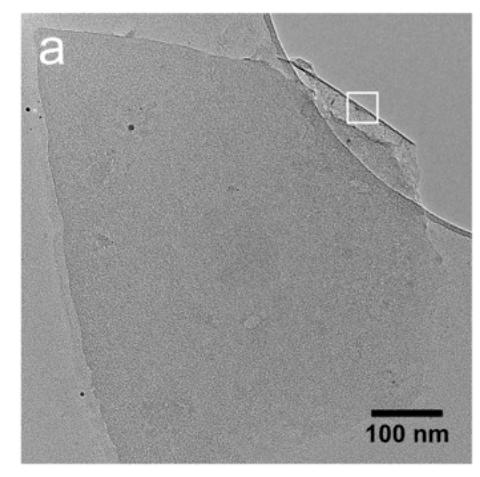

b
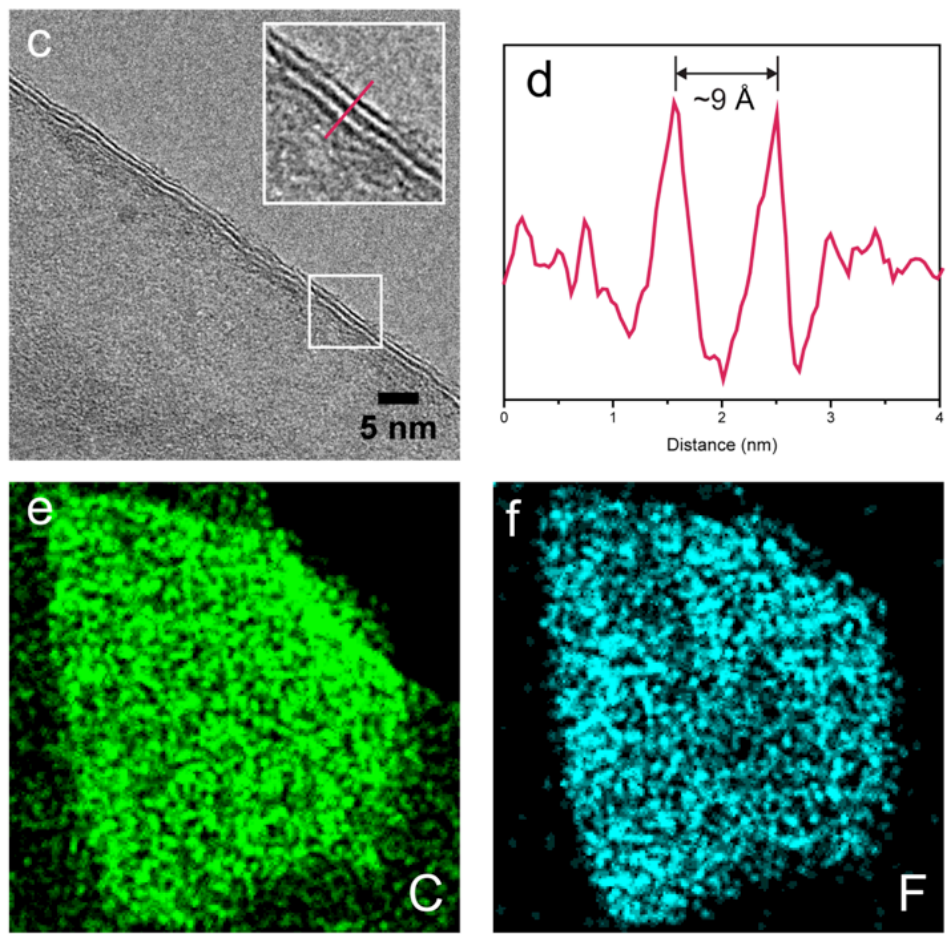

Fig. 4 (a) TEM image of an exfoliated $\left(\mathrm{C}_{2} \mathrm{~F}\right)_{\mathrm{n}}$ platelet, with (b) the corresponding SAED pattern. (c) Magnified TEM image from the indicated area in (a), showing the 
edge of the platelet. The inset in (c) shows a further magnified image from the edge. (d) A plot of the profile along the line in (c) inset, indicating a $\sim 0.9 \mathrm{~nm}$ interlayer distance. $(e, f)$ Elemental maps of $\mathrm{C}$ and $\mathrm{F}$ from the exfoliated $\left(\mathrm{C}_{2} \mathrm{~F}\right)_{\mathrm{n}}$ platelet.

Atomic force microscope (AFM) was used to examine the size and thickness of the exfoliated $\left(\mathrm{C}_{2} \mathrm{~F}\right)_{\mathrm{n}}$. Fig. 3a shows a typical image of the platelets from the chloroform dispersion. The particle size in lateral dimension shows a roughly 100 -fold decrease to 50-200 nm, which could result from exfoliation and/or centrifugation that removed larger flakes. AFM height analysis of the exfoliated $\left(\mathrm{C}_{2} \mathrm{~F}\right)_{\mathrm{n}}$ (Fig. 3b) suggests a layered structure, with a single layer roughly $0.9 \mathrm{~nm}$ in thickness.

Fig. 4a and $4 \mathrm{~b}$ show a transmission electron microscopy (TEM) image of an exfoliated platelet and its corresponding selected area electron diffraction (SAED) pattern that has a six-fold symmetry, indicating a hexagonal symmetry for the $\left(\mathrm{C}_{2} \mathrm{~F}\right)_{\mathrm{n}}$. A magnified TEM image (Fig. 4c), from the indicated area in Fig. 4a, shows the edge of the platelet, with a further zoomed-in image in the inset indicating 2-3 layers. Fig. 4d shows a plot of profile along the line in Fig. 4c inset, showing an interlayer distance of $\sim 0.9 \mathrm{~nm}$. The interlayer distance ranged from $0.8-0.9 \mathrm{~nm}$ along the lattice on the platelet edge. This value is consistent with the XRD and AFM results, and in good agreement with the proposed model. Elemental maps of carbon and fluorine acquired using a scanning transmission electron microscopy (STEM) mode indicate uniform presence of carbon and fluorine elements in the exfoliated $\left(\mathrm{C}_{2} \mathrm{~F}\right)_{\mathrm{n}}$. Of note is that the structure of $\left(\mathrm{C}_{2} \mathrm{~F}\right)_{\mathrm{n}}$ appeared to be sensitive to electron beam irradiation, which is evidenced by comparing the TEM images (Fig. S6) taken from the same area before and after short-term exposure to electron beam under TEM.

DFT calculations were used to investigate the stability and $\mathrm{C}-\mathrm{F}$ bonding of " $\mathrm{F}$ diamane"-like structure, as well as its mechanical and electronic properties. The structure of an unstrained monolayer is shown in Fig. 5. The calculated bond lengths and cells parameters for the unstrained $\left(\mathrm{C}_{2} \mathrm{~F}\right)$ monolayer are listed in Table $\mathbf{1}$. 


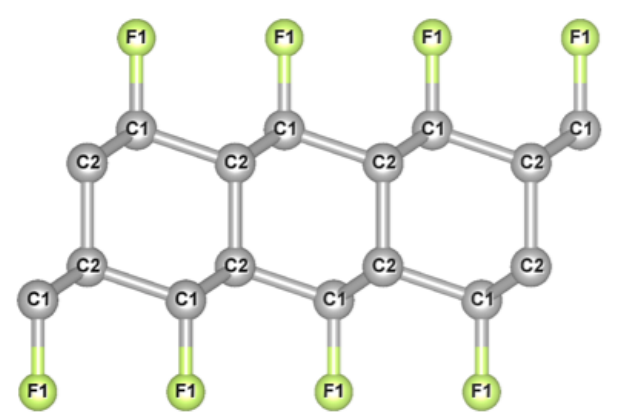

Fig. 5 Crystal structure of a "F-diamane"-like $\left(\mathrm{C}_{2} \mathrm{~F}\right)$ monolayer.

Table 1. Crystallographic description of the equilibrium $\left(\mathrm{C}_{2} \mathrm{~F}\right)$ monolayer.

\begin{tabular}{ccc}
\hline 2D-(C $\mathbf{2}$ F $)$ & Space Group & Atomic Position \\
\hline $\mathrm{V}_{\mathrm{o}}=219.5 \AA^{3}$ & $\mathrm{~N}^{\circ} 164 P-3 m 1$ & $\mathrm{C} 12 \mathrm{~d}(1 / 3,2 / 3,0.467)$ \\
$\mathrm{C} 2-\mathrm{C} 2=1.554 \AA$ & $a=b=2.559 \AA ; c=$ & $\mathrm{C} 22 \mathrm{c}(0.000,0.000$, \\
$\mathrm{C} 2-\mathrm{C} 1=1.561 \AA$ & $38.711 \AA$ & $0.520)$ \\
$\mathrm{C} 1-\mathrm{F} 1=1.378 \AA$ & $\alpha=\beta=90.00 \gamma=120.00$ & $\mathrm{~F} 12 \mathrm{~d}(1 / 3,2 / 3,0.431)$ \\
\hline
\end{tabular}

The results for the elastic stiffness verify the Born's mechanical stability criteria. The calculated values of the in plane Young modulus and Poisson ratio are $478.98 \mathrm{~N} \mathrm{~m}^{-1}$ and 0.10 , respectively. Based on the electronic band structure calculation, we found a direct band gap at the Gamma point $\left(4.02 \mathrm{eV}\right.$, see Fig. S7) for the $2 \mathrm{D}-\left(\mathrm{C}_{2} \mathrm{~F}\right)$ structure.

The local analysis of the electron density and its Laplacian at bond critical points (BCP) within the framework of the QTAIM method were used to investigate the covalency character of the chemical bonds of this structure. The $\mathrm{C}-\mathrm{C}$ bonds are characterized by higher electron density and larger negative Laplacian than the C-F bonds (see Table 2) indicating a typical covalent-shared interaction between the two C atoms, as seen from the ELF plots (see Fig. S8a). As shown in Fig. S8b, the higher electronegativity of fluorine shifts the $\mathrm{BCP}$ (along the $\mathrm{C}(1)-\mathrm{F}(1)$ ) path towards the $\mathrm{C}$ atom. A BCP was also found along the $\mathrm{F}(1)-\mathrm{F}(1)$ path, which is characterized by a very small value in both the electron density and the (positive) Laplacian. The C-F bonds, with a smaller positive value of the Laplacian, are interpreted as of partial ionic/covalent character. 
Table 2. Charge density $\rho(\mathrm{r})$ and its Laplacian $\nabla^{2} \rho(\mathrm{r})$ calculated at the BCP, in atomic units.

\begin{tabular}{ccc}
\hline Chemical meaning & $\boldsymbol{\rho}(\mathbf{r})$ & $\boldsymbol{\nabla}^{\mathbf{2}} \boldsymbol{\rho}(\mathbf{r})$ \\
\hline $\mathrm{C}(2)-\mathrm{C}(2)$ & 0.2329 & -0.5271 \\
$\mathrm{C}(2)-\mathrm{C}(1)$ & 0.2377 & -0.5401 \\
$\mathrm{C}(1)-\mathrm{F}(1)$ & 0.2575 & 0.1259 \\
$\mathrm{~F}(1)-\mathrm{F}(1)$ & 0.0148 & 0.0856 \\
\hline
\end{tabular}

A phonon dispersion curve of the $2 \mathrm{D}-\left(\mathrm{C}_{2} \mathrm{~F}\right)$ structure has been calculated using DFTP method. As shown in Fig. 6 no imaginary frequency appears indicating that the monolayer is stable at ambient condition.
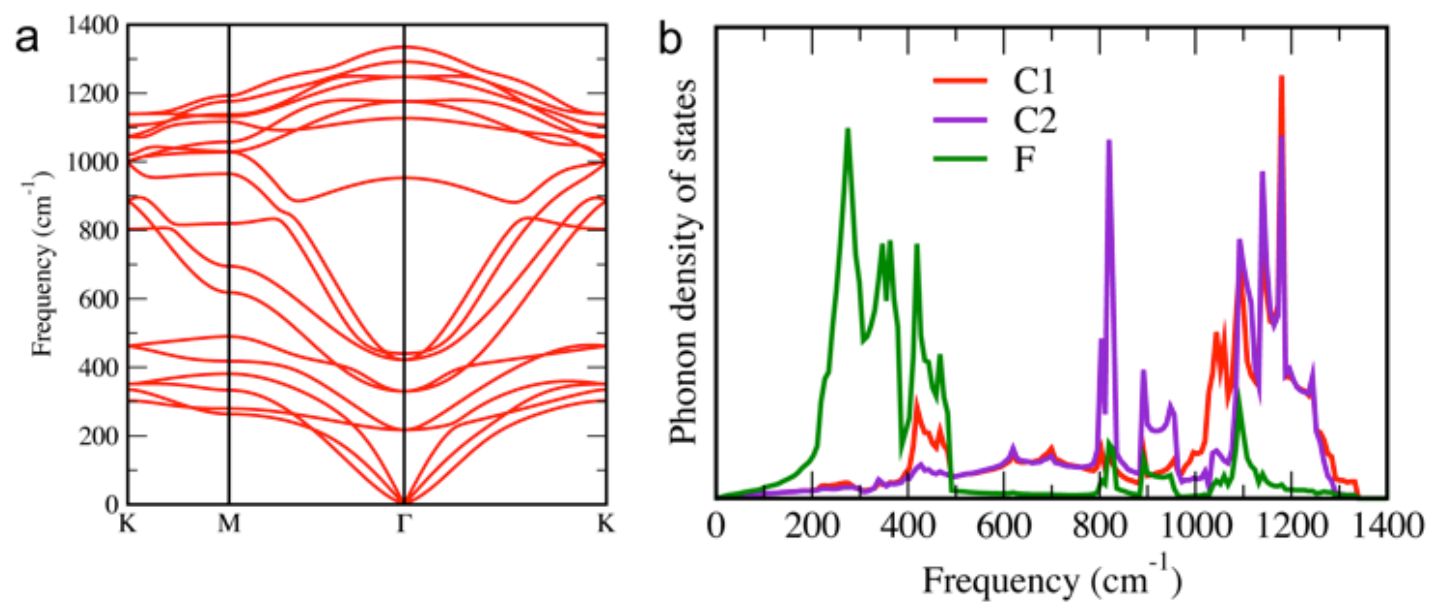

Fig. 6 Phonon dispersion curves of $2 \mathrm{D}-\left(\mathrm{C}_{2} \mathrm{~F}\right)(\mathrm{a})$ and vibrational DOS (b).

To complete the study of the vibrational properties we analysed the zone-centre phonons for the unstrained $\left(\mathrm{C}_{2} \mathrm{~F}\right)$ monolayer, Table 3. The mechanical representation yields the following multiplicities for the symmetry of the modes at the zone centre: $3 \mathrm{~A}_{1 \mathrm{~g}}+3 \mathrm{~A}_{2 \mathrm{u}}+3 \mathrm{E}_{\mathrm{u}}+3 \mathrm{E}_{\mathrm{g}}$, where the $\mathrm{A}_{2 \mathrm{u}}$ and $\mathrm{E}_{\mathrm{u}}$ modes are Infrared and Hyper-Raman active mode, and the $A_{1 g}$ and $E_{g}$ modes are active under Raman. The study of the vibrational properties of the $\left(\mathrm{C}_{2} \mathrm{~F}\right)$ monolayer confirms the experimental results on 
Raman and FTIR spectroscopy carried out in this work, showing an IR medium band at approximately $1300 \mathrm{~cm}^{-1}$.

In order to interpret the binding energies of the core levels measured by XPS, we performed DFT calculations for the $\mathrm{C} 1 \mathrm{~s}$ and $\mathrm{F} 1 \mathrm{~s}$ core states in the final state approximation. The calculated values of the binding energies are given in Table 4 . From these results we can see that the $\mathrm{C}$ atoms are bonded only to other $\mathrm{C}$ atoms $(\mathrm{C} 1 \mathrm{~s}-\mathrm{C})$ as the $\mathrm{C}-1 \mathrm{~s}$ values are smaller than if they were attached to fluorine $(\mathrm{C} 1 \mathrm{~s}-\mathrm{F})$, a similar situation as previously observed in a study on a $\mathrm{C}_{2} \mathrm{~F}-\mathrm{sp}^{3}$ slab supercell. ${ }^{[36]}$ The higher energy values of the core level obtained for the "F-diamane" monolayer can be related to the more covalent character of the C-F bonds than in the slab model (which is also supported by the calculated values of the Laplacian at the BCP). Our DFT results are in fair agreement with the experimental values of the core level.

Table 3. Calculated Raman- and Infrared-active phonon modes.

\begin{tabular}{ccc}
\hline Active modes & Raman $\left(\mathbf{c m}^{-\mathbf{1}}\right)$ & Infrared $\left(\mathbf{c m}^{\mathbf{- 1}}\right)$ \\
\hline $\mathrm{E}_{\mathrm{g}}$ & $216.92,421.63,1247.23$ & - \\
$\mathrm{A}_{1 \mathrm{~g}}$ & $440.34,1127.35,1292.19$ & - \\
$\mathrm{E}_{\mathrm{u}}$ & - & $329.66,1176.01$ \\
$\mathrm{~A}_{2 \mathrm{u}}$ & - & $915.07,1307.17$ \\
\hline
\end{tabular}

Table 4. Core level energies obtained in the DFT calculations. The C1s and F1s energy values are referred to the electrostatic potential at vacuum.

\begin{tabular}{cc}
\hline Core hole level site & Core level (eV) \\
\hline C1s (C) & 289.1 \\
C1s (F) & 290.5 \\
F1s (C) & 692.7 \\
\hline
\end{tabular}




\section{Conclusion}

We have demonstrated a strategy for preparing ultrathin "F-diamane"-like structures by fluorinating graphite to poly(dicarbon monofluoride) $\left(\mathrm{C}_{2} \mathrm{~F}\right)_{\mathrm{n}}$, followed by "topdown" exfoliation of $\left(\mathrm{C}_{2} \mathrm{~F}\right)_{\mathrm{n}}$ in solvents. Microscopic and spectroscopic analyses showed that the "F-diamane" structure was retained after exfoliation. The calculated topological parameters at the $\mathrm{BCP}$ suggest an increasing covalent character of the $\mathrm{C}-$ F bonds. This is related to higher energy values obtained for the core level, in good agreement with the experimental data. This method is suitable for preparing " $F$ diamane"-like materials in large quantity. Additionally, larger flakes of $\left(\mathrm{C}_{2} \mathrm{~F}\right)_{\mathrm{n}}$ could be made by fluorination of HOPG or graphite with larger flake size, which might allow a direct mechanical delamination of "F-diamane" single layers, e.g. using a sticky tape.

\section{Acknowledgements}

X.C. acknowledges the support from the Australian Research Council (DE180100294). S.R. acknowledges the support from MINECO Projects No. PID2019-106383GB-C43. C.Z. is grateful for the award of Future Fellowship from the Australian Research Council (FT170100224). The authors thank Xiao Wang for drawing the crystal structure model in Fig. 1. The authors thank the Mark Wainwright Analytical Centre at the University of New South Wales.

Keywords: Diamane $\bullet$ Graphite Fluoride $\bullet$ Fluorination $\bullet$ Exfoliation $\bullet$ 2D Materials

[1] L. A. Chernozatonskii, P. B. Sorokin, A. G. Kvashnin, D. G. Kvashnin, JETP Lett., 2009, 90, 134.

[2] M. A. Ribas, A. K. Singh, P. B. Sorokin, B. I. Yakobson, Nano Res., 2011, 4, 143.

[3] A. G. Kvashnin, L. A. Chernozatonskii, B. I. Yakobson, Nano Lett., 2014, 14, 676.

[4] L. Y. Antipina, P. B. Sorokin, J. Phys. Chem. C, 2015, 119, 2828.

[5] D. Odkhuu, D. Shin, R.S. Ruoff, N. Park, Sci. Rep., 2013, 3, 3276.

[6] D. C. Elias, R. R. Nair, T. M. G. Mohiuddin, S. V. Morozov, P. Blake, M. P. Halsall, A. C. Ferrari, D. W. Boukhavlov, M. I. Katsnelson, A. K. Geim, K. S. Novoselov, Science, 2009, 323, 610. 
[7] R. R. Nair, W. Ren, R. Jalil, I. Riaz, V. G. Kravets, L. Britnell, P. Blake, F. Schedin, A. S. Mayorov, S. Yuan, M. I. Katsnelson, H. M. Cheng, W. Strupinski, L. G. Bulusheva, A. V. Okotrub, I. V. Grigorieva, A. N. Grigorenko, K. S. Novoselov, A. K. Geim, Small, 2010, 6, 2877.

[8] M. Huang, P. V. Bakharev, Z. J. Wang, M. Biswal, Z. Yang, S. Jin, B. Wang, H. J. Park, Y. Li, D. Qu, Y. Kwon, X. Chen, S. H. Lee, M. G. Willinger, W. J. Yoo, Z. Lee, R. S. Ruoff, Nature Nanotechnol., 2020, 15, 289.

[9] P. V. Bakharev, M. Huang, M. Saxena, S. W. Lee, S. H. Joo, S. O. Park, J. Dong, D. C. Camacho-Mojica, S. Jin, Y. Kwon, M. Biswal, F. Ding, S. K. Kwak, Z. Lee, R. S. Ruoff, Nature Nanotechnol., 2020, 15, 59.

[10] S. Rajasekaran, F. Abild-Pedersen, H. Ogasawara, A. Nilsson, S. Kaya, Phys. Rev. Lett., 2013, 111, 085503.

[11] O. Ruff, O. Bretschneider, Z. Anorg. Allg. Chem., 1934, 217, 1.

[12] T. Nakajima, Fluorine-Carbon and Fluorine-Carbon Materials, Marcel Dekker, Inc., New York, 1995.

[13] Y. Kita, N. Watanabe, Y. Fujii, J. Am. Chem. Soc., 1979, 101, 3832.

[14] N. Watanabe, Solid State Ion., 1980, 1, 87.

[15] H. Touhara, K. Kadono, Y. Fujii, N. Watanabe, Z. Anorg. Allg. Chem., 1987, 544,7 .

[16] A. Tuteja, W. Choi, M. Ma, J. M. Mabry, S. A. Mazzella, G. C. Rutledge, G. H. McKinley, R. E. Cohen, Science, 2007, 318, 1618.

[17] Y. Hernandez, V. Nicolosi,M. Lotya, F.M. Blighe, Z. Sun, S. De, I. T. McGovern, B. Holland, M. Byrne, Y. K. Gun'ko, J. J. Boland, P. Niraj, G. Duesberg, S. Krishnamurthy, R. Goodhue, J. Hutchison, V. Scardaci, A. C. Ferrari, J. N. Coleman, Nat. Nanotechnol., 2008, 3, 563-568.

[18] X. Chen, J. F. Dobson, C. L. Raston, Chem. Commun., 2012, 48, 3703.

[19] X. Chen, R. A. Boulos, P. K. Eggers, C. L. Raston, Chem. Commun., 2012, 48, 11407.

[20] J. R. Brent, N. Savjani, E. A. Lewis, S. J. Haigh, D. J. Lewis, P. O’Brien, Chem. Commun., 2014, 50, 13338.

[21] R. Zbořil, F. Karlický, A. B. Bourlinos, T. A. Steriotis, A. K. Stubos, V. Georgakilas, K. Šafářová, D. Jančík, C. Trapalis, M. Otyepka, Small, 2010, 6, 2885. 
[22] Y. Yang, G. Lu, Y. Li, Z. Liu, X. Huang, ACS Appl. Mater. Interfaces, 2013, 5, 13478.

[23] M. Zhang, L. Liu, T. He, G. Wu, P. Chen, Mater. Lett., 2016, 171, 191.

[24] L. Liu, M. Zhang, Z. Xiong, D. Hu, G. Wu, P. Chen, Carbon, 2015, 81, 702.

[25] M. Dubois, K. Guérin, Y. Ahmad, N. Batisse, M. Mar, L. Frezet, W. Hourani, J. Bubendorff, J. Parmentier, S. Hajjar-Garreau, L. Simon, Carbon, 2014, 77, 688.

[26] M. Herraiz, M. Dubois, N. Batisse, S. Hajiar-Garreau, L. Simon, Dalton Trans., 2018, 47, 4596.

[27] S. Wu, J. Mo, Y. Zeng, Y. Wang, A. Rawal, J. Scott, Z. Su, W. Ren, S. Chen, K. Wang, W. Chen, Y. Zhang, C. Zhao, X. Chen, Small, 2019, 1903397.

[28] W. Feng, P. Long, Y. Feng, Y. Li, Adv. Sci., 2016, 3, 1500413.

[29] B. Wang, J. Wang, J. Zhu, ACS Nano, 2014, 8, 1862.

[30] V. Gupta, T. Nakajima, B. Žemva, J. Fluor. Chem., 2001, 110, 145.

[31] W. Rüdorff, K. Brodersen, Z. Naturforsch. B, 1957, 12, 595.

[32] R. J. Lagow, R. B. Badachhape, J. L. Wood, J. L. Margrave, J. Chem. Soc., Dalton Trans., 1974, 1268.

[33] W. Zhang, M. Dubois, K. Guérin, A. Hamwi, J. Giraudet, F. Masin, J. Solid State Chem., 2008, 181, 1915.

[34] M. Dubois, J. Giraudet, K. Guérin, A. Hamwi, Z. Fawal, P. Piotte, F. Masin, J. Phys. Chem. B, 2006, 110, 11800.

[35] M. Herraiz, N. Batisse, M. Dubois, V. V. Nesvizhevsky, C. Cavallari, M. Brunelli, V. Pischedda, S. Radescu, J. Phys. Chem. C, 2020, 124, 14229.

[36] C. Cavallari, S. Radescu, M. Dubois, N. Batisse, H. Diaf, V. Pischedda, J. Phys. Chem. C, (in press) doi: 10.1021/acs.jpcc.0c06860. 


\section{Supporting Information}

\section{Liquid-Phase Exfoliation of Poly(Dicarbon Monofluoride) $\left(C_{2} F\right)_{n}$}

Xianjue Chen, ${ }^{\mathrm{a}, *}$ Marc Dubois,,${ }^{\mathrm{b}}{ }^{*}$ Silvana Radescu, ${ }^{\mathrm{c}}$ Aditya Rawal, ${ }^{\mathrm{d}}$ Chuan Zhao ${ }^{\mathrm{a}}$

${ }^{a}$ School of Chemistry, University of New South Wales, Sydney, New South Wales 2052, Australia,

${ }^{b}$ Université Clermont Auvergne, SIGMA Clermont, CNRS, Institut de Chimie de Clermont-Ferrand (UMR 6296), BP 10448, F-63000, Clermont-Ferrand, France

${ }^{c}$ Departamento de Física, Universidad de La Laguna, Instituto de Materiales y Nanotecnología, 38200 La Laguna S/C Tenerife, Spain

${ }^{d}$ Mark Wainwright Analytical Centre, University of New South Wales, Sydney, New South Wales 2052, Australia

*Email: correspondence and requests for materials should be addressed to X.C. (xianjue.chen@unsw.edu.au) and M.D. (marc.dubois@uca.fr) 


\section{Experimental details:}

The $\left(\mathrm{C}_{2} \mathrm{~F}\right)_{\mathrm{n}}$ was synthesized by heating graphite flakes to $350{ }^{\circ} \mathrm{C}$ under pure $\mathrm{F}_{2}$ gas flow. (CF) $($ GT1FF012) were purchased from ACS Material and used without further modification. Suspensions $(10 \mathrm{~mL})$ of $\left(\mathrm{C}_{2} \mathrm{~F}\right)_{\mathrm{n}}\left(0.5 \mathrm{mg} \mathrm{mL} \mathrm{mL}^{-1}\right)$ in water or organic solvents were subject to sonication using a probe-sonicator (Branson Digital Sonifier 450; 20\% amplitude, $1 \mathrm{~h}$; under ice bath and with a pulse effect of $10 \mathrm{~s}$ on and $10 \mathrm{~s}$ off to avoid overheating). The suspensions in organic solvents were centrifuged (Centrifuge 5810, Eppendorf, $117 \times g, 10 \mathrm{~min}$ ) to remove non-dispersible species. The supernatants were separated affording stable suspensions. XRD patterns were obtained using a PANalytical Xpert Multipurpose X-ray Diffraction System and analyzed using X'Pert HighScore Plus software. SEM images were recorded on a FEI Nova NanoSEM 450 FE-SEM. XPS spectra were acquired using an ESCALAB 250 $\mathrm{Xi}$, Thermo Scientific. The ${ }^{19} \mathrm{~F}$ solid-state NMR spectra were acquired on a Bruker AVANCE III $700 \mathrm{MHz}$ spectrometer with a 16.4 tesla superconducting magnet, operating at a frequency of $658.88 \mathrm{MHz}$ for the ${ }^{19} \mathrm{~F}$ nucleus. Approximately $30 \mathrm{mg}$ of sample was packed in a in $3.2 \mathrm{~mm}$ zirconia rotors fitted with Vespel® caps and spun to $24 \mathrm{kHz}$ at the magic angle in a double resonance $3.2 \mathrm{~mm} \mathrm{HX}$ Low-gamma MAS probehead. The ${ }^{19} \mathrm{~F} 90^{\circ}$ pulse length of $2.5 \mu$ s was used with sufficient recycle delay to ensure full signal relaxation. The ${ }^{19} \mathrm{~F}$ chemical shifts were referenced to $\mathrm{CFCl}_{3}$ at 0 ppm using the ${ }^{19} \mathrm{~F}$ signal of polytetrafluoroethylene (PTFE) at $-122.7 \mathrm{ppm}$ as a secondary reference. The DMFIT software was used for deconvolution of the NMR spectra ${ }^{[1]}$ AFM images were acquired on a Bruker Dimension ICON SPM operating in the tapping mode. A dispersion of $\left(\mathrm{C}_{2} \mathrm{~F}\right)_{\mathrm{n}}$ in chloroform was drop-cast onto a freshly cleaved mica substrate and dried in air. AFM images were analyzed using the Gwyddion SPM software. TEM specimens were prepared by drop casting a $\left(\mathrm{C}_{2} \mathrm{~F}\right)_{\mathrm{n}}$ dispersion onto a holey carbon-coated $\mathrm{Cu}$ grid (SPI Supplies, \#2450-AB) and dried in air. TEM/STEM analysis was performed using a JEOL JEM-F200 Multi-Purpose FEG-S/TEM operating at an accelerating voltage of $80 \mathrm{kV}$. Image $\mathrm{J}$ and Pathfinder were used for processing the TEM and elemental mapping images. Raman spectra were recorded on a Renishaw InVia 2 Raman spectrometer at room temperature with a $532 \mathrm{~nm}$ laser excitation. For FTIR, pellets were prepared by compressing the wellmixed $\mathrm{KBr}$ and sample powders. FTIR was recorded on a Spectrum 100/Spotlight 400 spectrometer in the transmission mode. 


\section{Computational details:}

The DFT calculations for the $\left(\mathrm{C}_{2} \mathrm{~F}\right)$ monolayer were performed using the Vienna $\mathrm{Ab}$ Initio Simulation Package (VASP) computational code, which implements the projector-augmented wave method (PAW), and the Perdew-Burke-Ernzerhof (PBE) form of the generalized gradient approximation was taken for the exchange and correlation (XC) energy. ${ }^{[2]}$ A large vacuum space between monolayers of $33.5 \AA$ is set to avoid interaction between the periodic images of the layers. In the ab initio calculations, only the two $2 \mathrm{~s}$ and two $2 \mathrm{p}$ outermost valence electrons of $\mathrm{C}$ and the two $2 \mathrm{~s}$ and five $2 \mathrm{p}$ outermost valence electrons of $\mathrm{F}$ were explicitly taken into account, the rest of the electrons being considered frozen at the core. To ensure a convergence in the energy differences between phases of around $1 \mathrm{meV}$ we used dense uniform $15 \times 15 \times 1$ k-points Monkhorst-Pack grids to sample the Brillouin zone, and a cutoff in the kinetic energy of the plane-waves basis set of $600 \mathrm{eV}$. To obtain the unstrained configuration, the atomic positions and lattice vectors were fully relaxed (with residual forces less that $0.005 \mathrm{eV} \AA^{-1}$ ) and the optimized (relaxed) coordinates were then used for the electronic, elastic, vibrational properties and core level binding energies.

The mechanical stability of the unstrained monolayer was checked in terms of elastic constants (Born stability criteria). In VASP calculations, the elastic constants for 2D materials can be obtained by normalizing the calculated constant with the lattice parameter c. By applying a set of suitable deformations to the monolayer and taking into account the reduced dimensionality, we obtained the in-plane elastic constants, where the Young's modulus (E) and Poisson's ratio (v) can be obtained from the following relationships ${ }^{[3]}$ :

$E=\left(C_{11}^{2}-C_{12}^{2}\right) / C_{11} \quad v=\frac{C_{12}}{C_{11}}$

To check the stability of $2 \mathrm{D}-\left(\mathrm{C}_{2} \mathrm{~F}\right)$ we have calculated the phonon dispersion curves in the whole BZ zone (a structure is locally stable only if the calculated frequencies of all its phonon modes are positive; otherwise, the presence of negative (imaginary) frequencies indicates an instability). In order to calculate the phonons we used the 
density functional perturbation theory (DFPT) as implemented in the VASP code and a post processing Phonopy package to analyze the vibrational modes. ${ }^{[4]}$

The electron localization function (ELF) was calculated from the valence electronic density obtained within the PAW scheme implemented in VASP (where the core electrons are considered frozen and not explicitly considered). The visualization of the ELF isosurface was done with VESTA 3.5.5. ${ }^{[5]}$ We further performed a topological analysis of the all-electron density (the sum of the core and valence densities) calculated with VASP by means of the quantum theory of atoms in molecules (QTAIM), using the CRITIC2 code. ${ }^{[6]}$ For the calculation of the core level binding energies of the $\mathrm{C} 1 \mathrm{~s}$ and $\mathrm{F} 1 \mathrm{~s}$ core state with a half core-hole effects we have applied the so-called final state method based in DFT. ${ }^{[7]}$ 

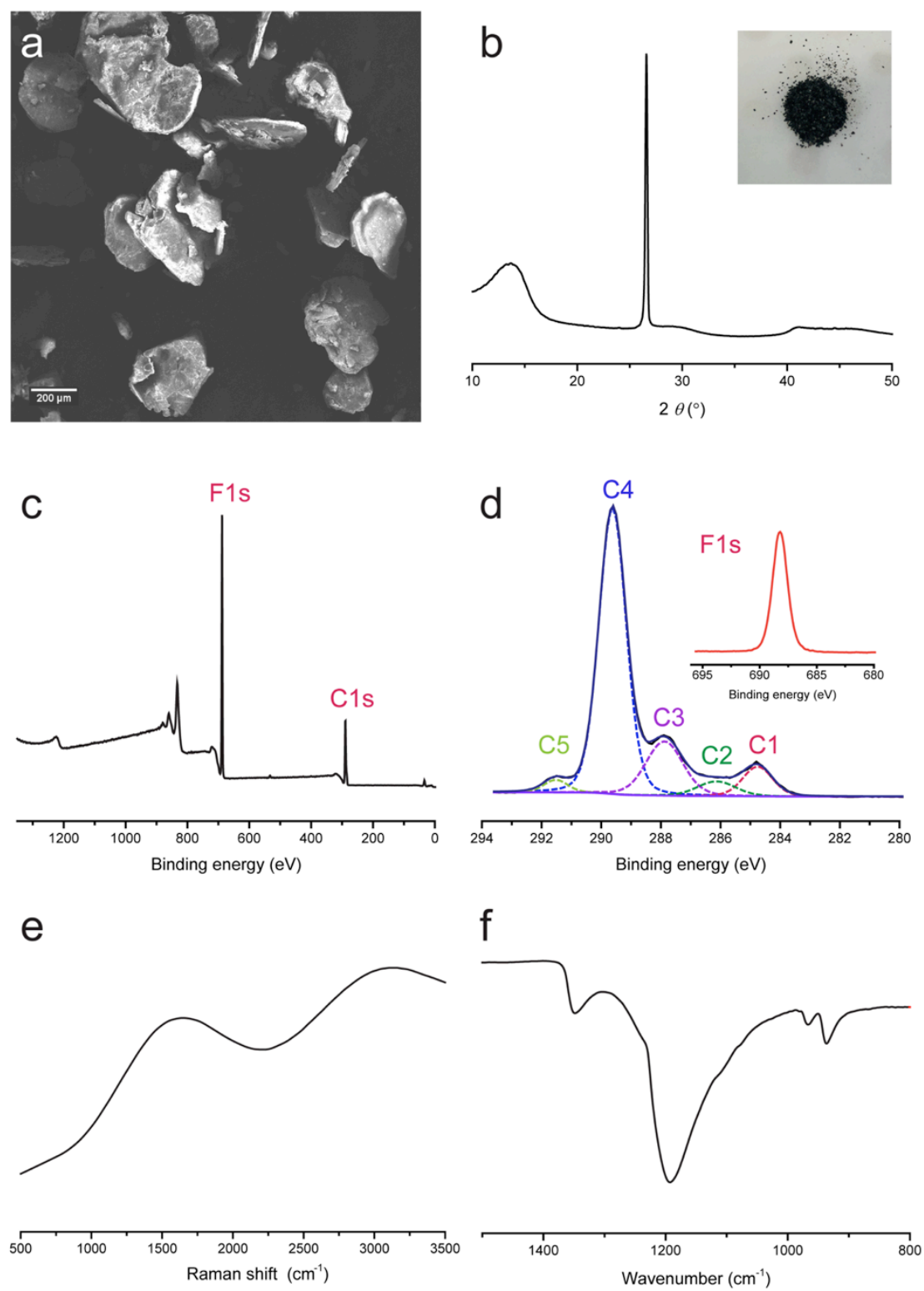

Fig. S1 (a) SEM image, (b) XRD pattern, (c) XPS survey, (d) C1s and F1s spectra, (e) Raman spectrum, and (f) FTIR spectrum of $(\mathrm{CF})_{\mathrm{n}}$. 


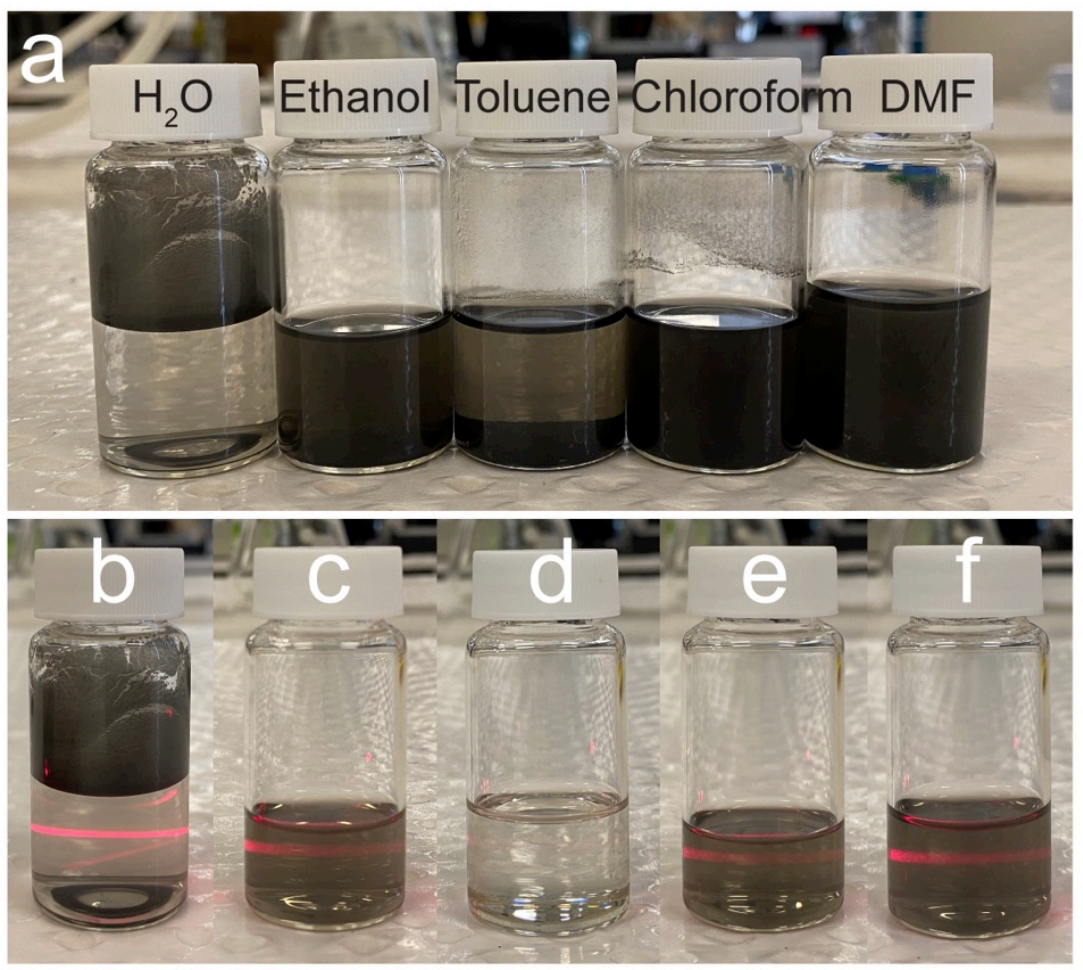

Fig. S2 (a) Photograph of $\left(\mathrm{C}_{2} \mathrm{~F}\right)_{\mathrm{n}}$ dispersed in different solvents through ultrasonication. (b-f) Tyndall effect confirming the colloidal nature of $\left(\mathrm{C}_{2} \mathrm{~F}\right)_{\mathrm{n}}$ dispersed in solvents, (b) water, (c) ethanol, (d) toluene, (e) chloroform, and (f) DMF. The dispersions in (c-f) were the supernatants after centrifugation $(117 \times g, 10 \mathrm{~min})$. 

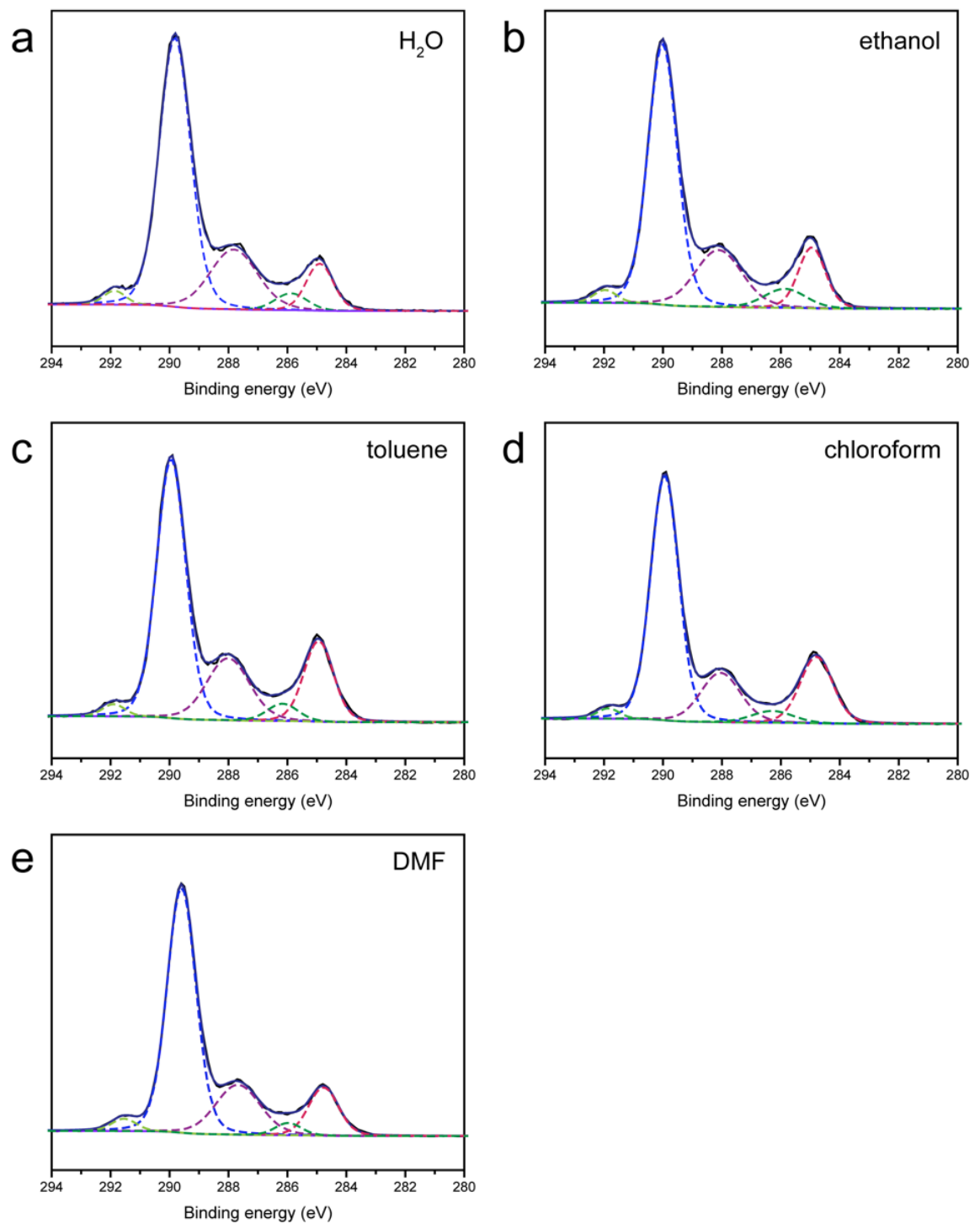

Fig. $\mathbf{S 3}$ XPS C1s spectra of exfoliated $\left(\mathrm{C}_{2} \mathrm{~F}\right)_{\mathrm{n}}$ in different solvents. 


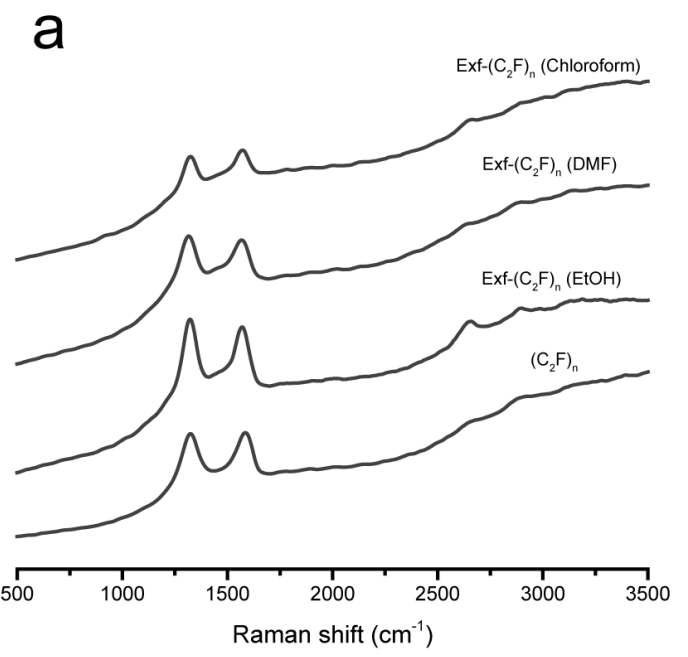

b

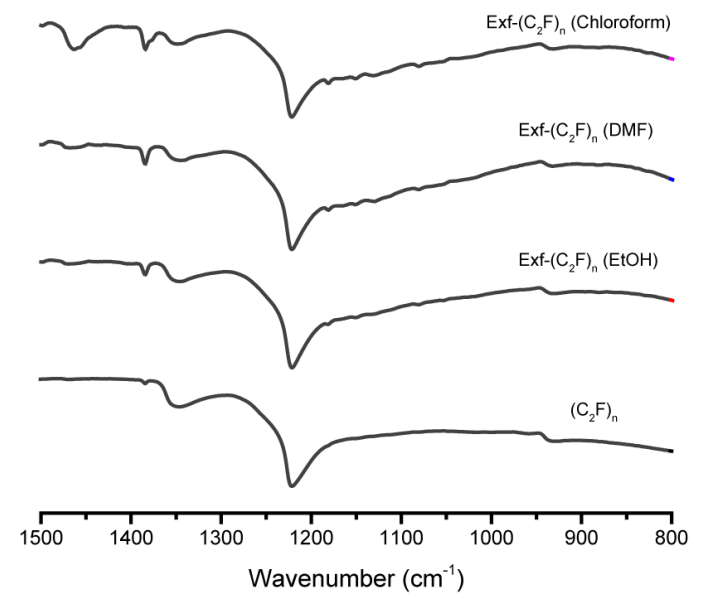

Fig. S4 (a) Raman and (b) FTIR spectra of $\left(\mathrm{C}_{2} \mathrm{~F}\right)_{\mathrm{n}}$ before and after exfoliation in different solvents. 

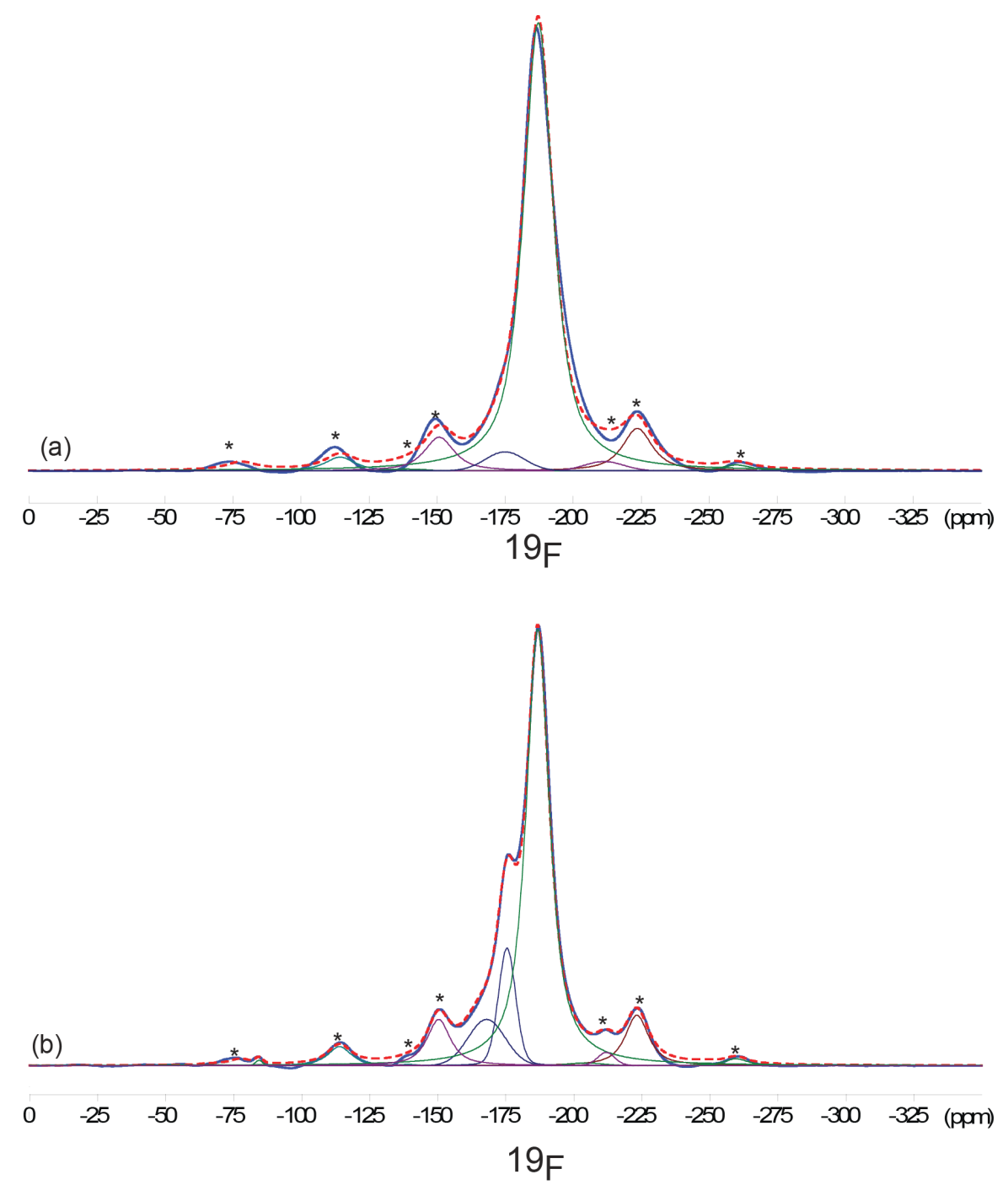

Fig. S5 Deconvolution of the center band and spinning sidebands of the ${ }^{19} \mathrm{~F}$ solid state NMR spectra of (a) $(\mathrm{CF})_{\mathrm{n}}$ and $(\mathrm{b})\left(\mathrm{C}_{2} \mathrm{~F}\right)_{\mathrm{n}}$. The bold solid line in blue is the experimental spectrum, the bold dashed line in red is the overall fit to the spectrum and the individual components of the fit are plotted as thin solid lines. The spinning sidebands are marked by $*$. The relative fractions of the different fluorine species are presented below.

\begin{tabular}{|c|c|c|}
\hline Sample & $\begin{array}{c}\text { Position } \\
(\mathrm{ppm})\end{array}$ & $\begin{array}{c}\text { Relative } \\
\text { fraction }(\%)\end{array}$ \\
\hline \multirow{3}{*}{$\left(\mathrm{C}_{2} \mathrm{~F}\right)_{\mathrm{n}}$} & -187 & 80.6 \\
\cline { 2 - 3 } & -175 & 11.0 \\
\cline { 2 - 3 } & -168 & 0.2 \\
\cline { 2 - 3 } & -84 & 8.2 \\
\hline \multirow{7}{*}{$(\mathrm{CF})_{\mathrm{n}}$} & -186 & 95.0 \\
\cline { 2 - 3 } & -175 & 5.0 \\
\hline
\end{tabular}



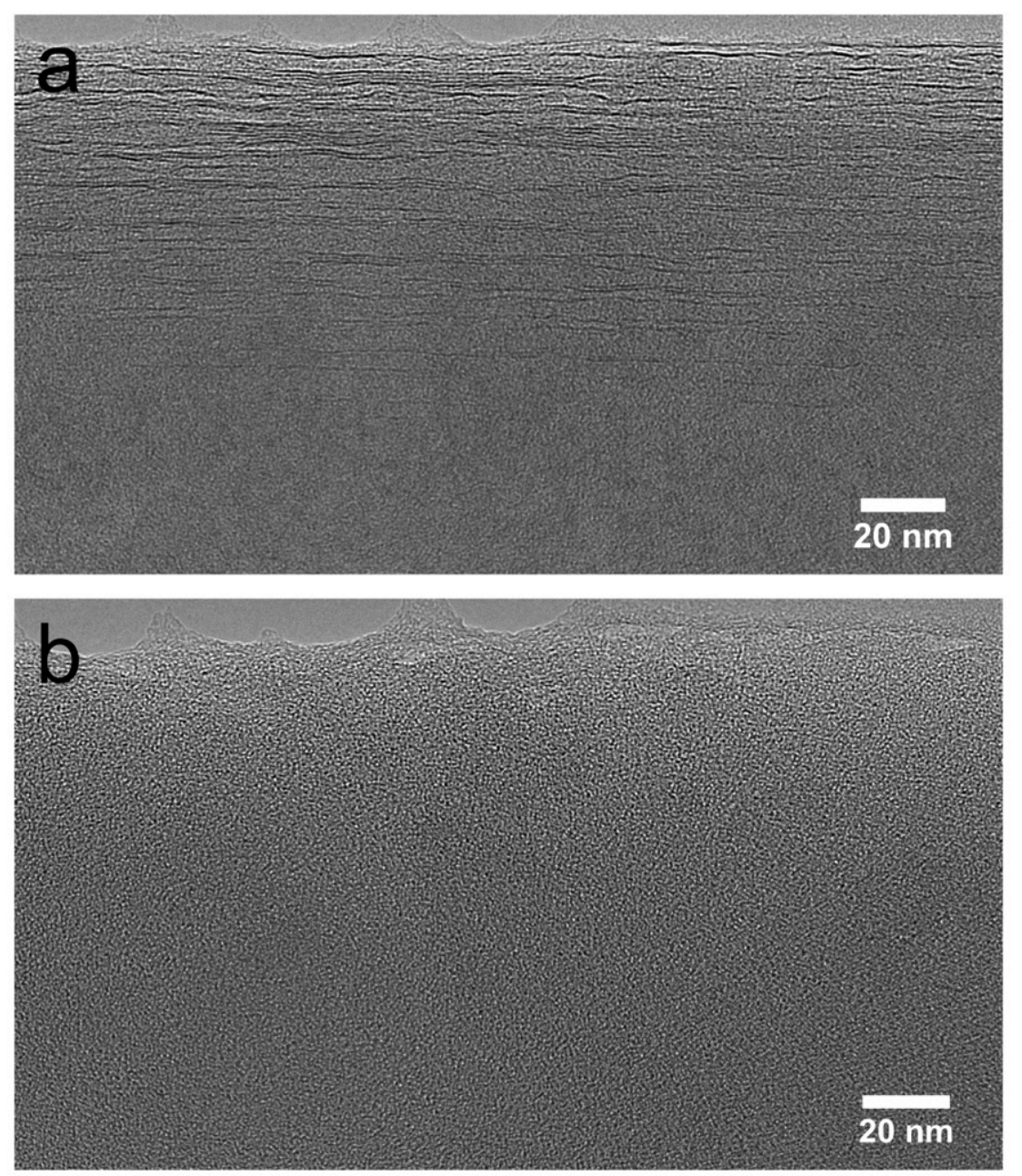

Fig. S6 TEM images of a $\left(\mathrm{C}_{2} \mathrm{~F}\right)_{\mathrm{n}}$ flake (a) before and (b) after 2-minute exposure to electron beam under TEM. 


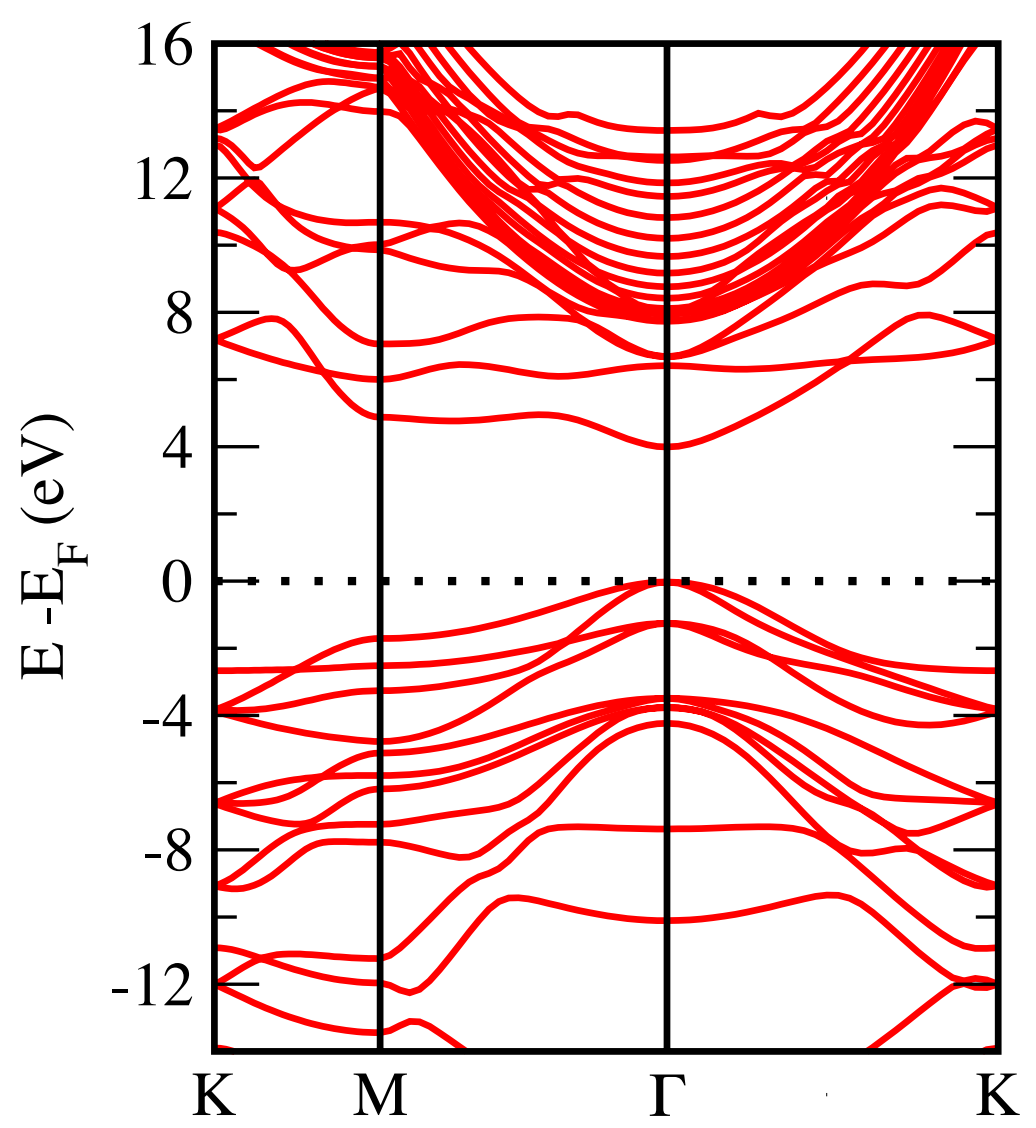

Fig. $\mathbf{S} 7$ Band structure of $2 \mathrm{D}-\left(\mathrm{C}_{2} \mathrm{~F}\right)$.
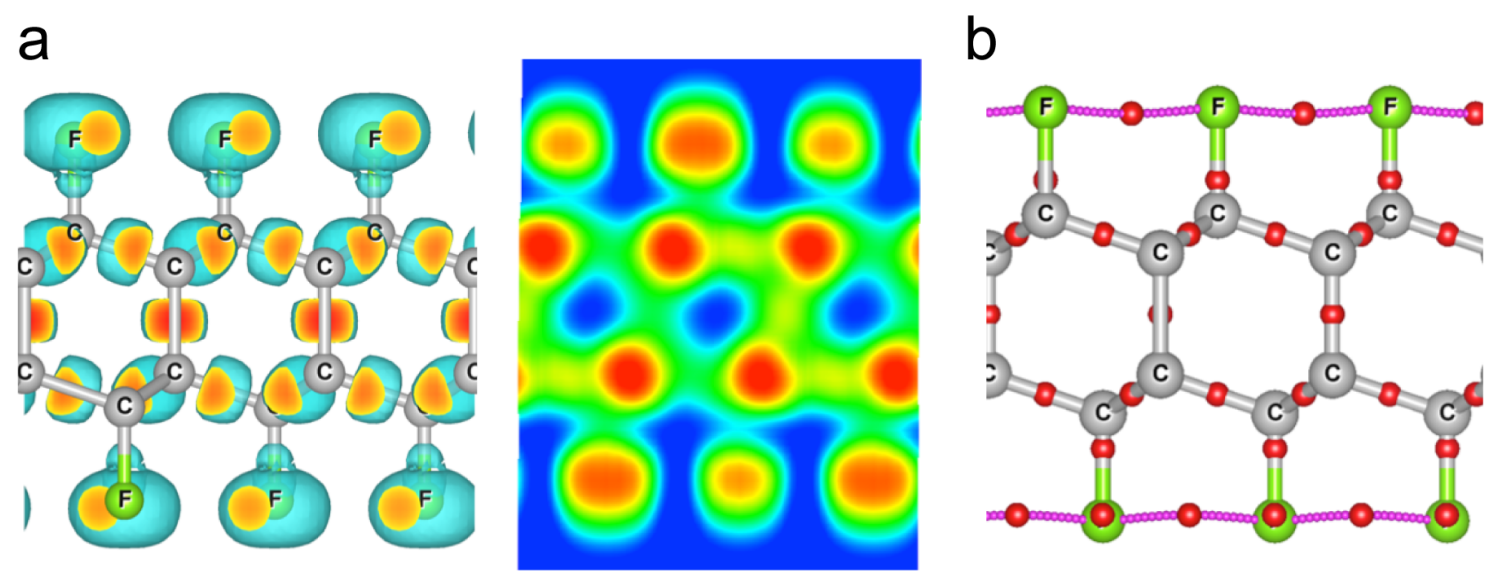

Fig. S8 (a) The ELF isosurface (0.72) shown in different projections, with red regions corresponding to maxima in ELF. (b) the bond critical points (BCP) shown as red circle and the violet lines showing the bond paths. 
Reference

1. D. Massiot, F Fayon, M. Capron, I. King, S. Le Calvé, B. Alonso, J. O. Durand, B. Bujoli, Z. Gan, G. Hoatson, Magn. Reson. Chem., 2002, 40, 70.

2. G. Kresse, J. Furthmüller, Comput. Mater. Sci., 1996, 6, 15-50; G. Kresse, J. Furthmüller, Phys. Rev. B, 1996, 54, 11169-11186; J. P. Perdew, A. Ruzsinszky, G. I. Csonka, O. A. Vydrov, G. E. Scuseria, L. A. Constantin, X. Zhou, K. Burke, Phys. Rev. Lett., 2008, 100, 13; J. P. Perdew, K. Burke, M. Ernzerhof, Phys. Rev. Lett., 1996, 77, 3865-3868.

3. L. Vannucci, U. Petralanda1, A. Rasmussen, T. Olsen, K. S. Thygesen, J. Appl. Phys., 2020, 128, 105101.

4. A. Togo, I. Tanaka, Scr. Mater., 2015, 108, 1-5..

5. K. Momma, F. Izumi, J. Appl. Crystallography, 2011, 44, 1272-1276.

6. R. F. W. Bader, Atoms in Molecules: A Quantum Theory. Oxford, New York: Oxford University Press, 1994; A. Otero-de-la-Roza, E. R. Johnson, V. Luaña, Comput. Phys. Commun., 2014, 185, 1007-1018.

7. L. Köhler, G. Kresse, Phys. Rev. B, 2004, 70, 165405. 